\title{
Images in Anesthesia: A right atrial foreign body
}

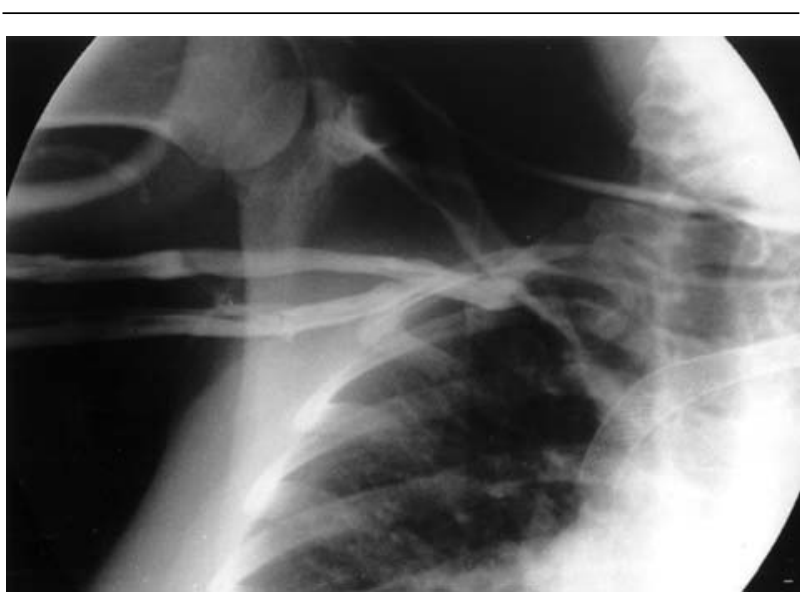

FIGURE 1 Venography shows a detached piece of stent in the right atrium which is fractured from the stent in the left subclavian vein.

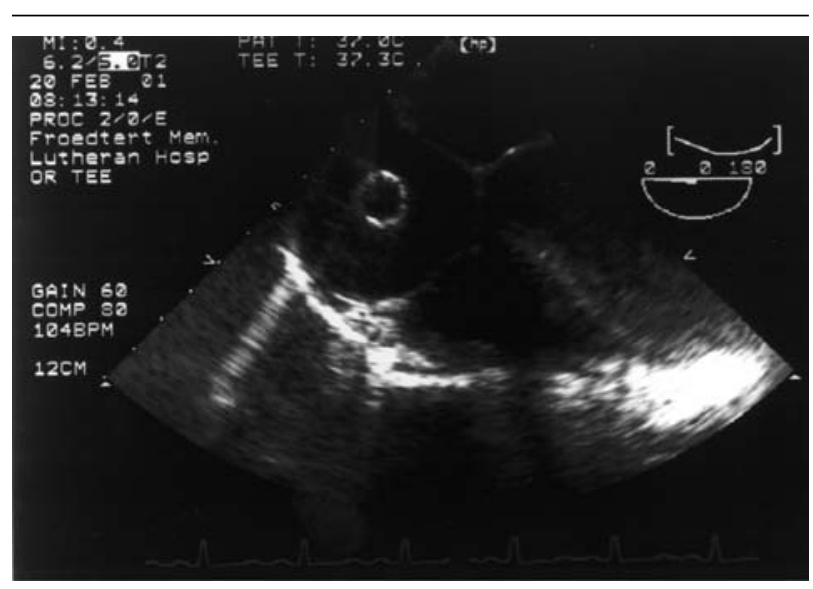

FIGURE 2 Intraoperative transesophageal echocardiography shows the stent in the right atrium.
A 27-yr-old male patient on hemodialysis for end stage renal disease presented for surgical removal of a fractured A-V fistular stent, which had migrated from the left upper extremity fistula site to the right atrium. A polytetrafluoroethylene loop graft had been placed in the left arm in 1999 with revision a year later. During hemodialysis, an increase in the venous pressure was observed and a venogram was performed. It revealed the stent overlapping the occluded left subclavian, axillary and brachiocephalic veins with a portion of the stent extending into the superior vena cava (SVC) and the right atrium (RA; Figure 1). Preoperative transesophageal echocardiography revealed a tubular structure $1.7 \mathrm{~cm}$ in diameter in the SVC and extending into the RA abutting its lateral aspect below the tricuspid valve (Figure 2). No thrombus was noted. On cardiopulmonary bypass a right atriotomy was performed and findings were that of severe fibrosis over the innominate vein and a fractured stent located in the SVC with a distally detached portion extending into the RA and inferior vena cava. The stent was a 7 $\mathrm{cm}$ long wire mesh with a $10-\mathrm{cm}$ long inner silicone catheter. The stent was removed from the RA.
Migration of a dialysis fistula stent to the atrium is a rare complication. ${ }^{1-3}$ They are dangerous as they might migrate to the right ventricle, embolize in the pulmonary tree or serve as a source of recurrent pulmonary emboli. ${ }^{2}$

Lei Yao MD PhD

Anna Maria Veytsman MD

M. Saeed Dhamee MD

Milwaukee, Wisconsin

\section{References}

1 Amaouche A. Stent migration in a hemodialysis patient (French). Nephrologie 2001; 22: 47.

2 Schillinger F, Montagnac R, Milccnt T, Chancrin JP, Mahmandar A, Pernet P. Pulmonary migration of an axillary stent in a chronic hemodialysis patient (French). Nephrologie 2001; 22: 49-51.

3 Felner JM, Churchwell AL, Murphy DA. Right atrial thromboemboli: clinical, echocardiographic and pathophysiologic manifestations. J Am Coll Cardiol 1984; 4: 1041-51. 